\title{
D’une conception épistémologique d'une discipline scolaire à sa mise en œuvre dans la classe : quelles difficultés pour un professeur débutant?
}

L'exemple de la définition de la population active en sciences économiques et sociales (classe de Seconde)

From an epistemological conception of a school discipline to its implementation in the classroom: what difficulties for a beginning teacher? The example of the definition of the active population in economic and social sciences (french class of Second)

\section{Christine Dollo}

\section{OpenEdition Journals}

Édition électronique

URL : https://journals.openedition.org/educationdidactique/2946

DOI : 10.4000/educationdidactique.2946

ISSN : 2111-4838

\section{Éditeur}

Presses universitaires de Rennes

\section{Édition imprimée}

Date de publication : 28 août 2018

Pagination : 43-58

ISBN : 978-2-7535-7554-7

ISSN : 1956-3485

\section{Référence électronique}

Christine Dollo, « D'une conception épistémologique d'une discipline scolaire à sa mise en œuvre dans la classe : quelles difficultés pour un professeur débutant? », Éducation et didactique [En ligne], 12-1 | 2018, mis en ligne le 28 août 2020, consulté le 16 août 2022. URL : http://journals.openedition.org/ educationdidactique/2946; DOI : https://doi.org/10.4000/educationdidactique.2946 


\title{
D'UNE CONCEPTION ÉPISTÉMOLOGIQUE D'UNE DISCIPLINE SCOLAIRE À SA MISE EN GUVRE DANS LA CLASSE : QUELLES DIFFICULTÉS POUR UN PROFESSEUR DÉBUTANT ? L'EXEMPLE DE LA DÉFINITION DE LA POPULATION ACTIVE EN SCIENCES ÉCONOMIQUES ET SOCIALES (CLASSE DE SECONDE)
}

\author{
Christine Dollo \\ Aix-Marseille université, ADEF, Marseille, France
}

\begin{abstract}
Lobjectif de cet article est de mettre en évidence la difficulté de passage d'une certaine conception épistémologique des sciences économiques et sociales (fondée sur la rupture avec le sens commun) à des scénarios pédagogiques permettant la mise en œuvre, par des professeurs débutants, de démarches didactiques s'appuyant sur cette épistémologie de la discipline. Après avoir présenté une conception de la discipline s'opposant à l'inductivisme et aux « fondamentaux », le texte explore certaines difficultés des professeurs débutants sur la base d'une étude de cas. Il s'inscrit ainsi dans le cadre d'une « recherche de faisabilité ». L'expérimentation présentée concerne la définition du concept de population active, prélude à une étude du phénomène du chômage, en classe de Seconde.
\end{abstract}

Mots-clés : démarche d'investigation, démarche scientifique, didactique des sciences économiques et sociales, épistémologie, formation des professeurs débutants, représentations sociales.

From an epistemological conception of a school discipline to its implementation in the classroom: what difficulties for a beginning teacher? The example of the definition of the active population in economic and social sciences (french class of Second)

The objective of this article is to highlight the difficulty of moving from a certain epistemological conception of the economic and social sciences (based on the break with common sense) to pedagogical scenarios allowing the implementation, by beginning teachers, of didactic approaches based on this epistemology of the discipline. After presenting a conception of the discipline opposing inductivism and "fundamentals", the text explores some difficulties of beginning teachers based on a case study. It is thus part of a "feasibility research." The experimentation presented concerns the definition of the concept of the active population, a prelude to a study of the phenomenon of unemployment in the French class of Second.

Keywords: investigative approach, scientific approach, didactics of economic and social sciences, epistemology, training of beginning teachers, social representations.

Remerciements :

Je remercie les experts anonymes du comité de lecture de la revue pour les commentaires instructifs et éclairants qu'ils ont fournis sur la version initiale de ce texte. Le propos final n'engage évidemment que son auteure. 


\section{INTRODUCTION}

Depuis la création des IUFM (aujourd'hui ESPE), et parallèlement au développement de travaux en didactique des sciences économiques et sociales (SES), nous avons défendu, dans le cadre notamment de la formation professionnelle des enseignants de SES, une conception épistémologique de la discipline fondée sur la rupture avec le sens commun (Dollo, 2009). Nous avons ainsi montré (Dollo, 2005a) que la discipline scolaire SES ne pouvait plus se justifier par ses méthodes (pédagogies actives, travail sur documents, etc.), largement utilisées dans le système scolaire, mais qu'elle devait le faire, comme toutes les disciplines scolaires, à partir des savoirs qu'elle se propose de transmettre aux élèves. Elle ne doit ainsi plus constituer, selon une expression d'Yves Chevallard, une " anomalie didactique " (1997). Ces savoirs ont cependant des particularités, ils renvoient à des débats de société (chômage, mondialisation, exclusion, etc.) et il importe de bien situer la spécificité d'une approche scolaire de ces questions, ce qui suppose que l'on ne confonde pas leur étude scientifique et le débat médiatique ou politique relatif à ces mêmes questions (Dollo, 2009).

Les élèves ont en effet souvent une conception positiviste de "la science » qu'ils assimilent aux sciences de la nature, ce qui constitue un obstacle à la prise en compte du caractère scientifique des propositions élaborées par les sciences sociales. On trouve ainsi chez les élèves un modèle épistémologique implicite selon lequel il existerait une réponse unique à tout problème scientifique (comme, selon eux, en sciences physiques par exemple, même si on sait bien que la physique savante est elle-même traversée par des paradigmes concurrents). Les disciplines de référence des sciences économiques et sociales ne seraient alors pas des sciences puisqu'elles présentent plusieurs réponses à certains problèmes, différentes selon le paradigme considéré, la science économique (notamment) étant en effet une science dite pluriparadigmatique (Dollo, 2003). Un risque existe alors : pour les élèves, les différents thèmes abordés ne sont finalement qu'une "affaire d'opinion». Et si tel sujet est affaire d'opinions, pourquoi l'une d'elles (celle du professeur par exemple) serait-elle « supérieure » à la leur?

Nous adhérons cependant au discours de Gaston Bachelard, pour qui « La science, dans son besoin d'achèvement comme dans son principe, s'op- pose absolument à l'opinion [...]. On ne peut rien fonder sur l'opinion : il faut d'abord la détruire. Elle est le premier obstacle à surmonter » (Bachelard, 1983, p. 14). De fait, " les travaux sur l'apprentissage montrent que le rapport aux savoirs des élèves en difficulté scolaire tend à relativiser les savoirs scolaires au nom d'une "vérité" de l'expérience et que cette attitude empêche précisément d'entrer dans une posture d'apprentissage » (Deauviau, 2007, p. 108). Il est donc important « de conduire les élèves à transformer progressivement leurs conceptions épistémologiques afin d'adopter une posture plus cohérente avec les acquis de l'épistémologie savante » (Beitone, Dollo, Hemdane, Lambert, 2013, p. 61).

Les évolutions curriculaires semblent aujourd'hui conforter cette conception épistémologique puisque, les programmes de SES mis en place depuis les rentrées 2010 pour la classe de Seconde, 2011 et 2012 pour les classes de Première et Terminale ES (avec un aménagement apporté en 2013), insistent sur le fait que « l'enseignement des sciences économiques et sociales au lycée vise à former les élèves à une posture intellectuelle, celle du rationalisme critique et de la vigilance épistémologique. Il s'agit pour eux d'apprendre à porter un regard savant sur le monde social et par là de former leur esprit à prendre du recul par rapport aux discours médiatiques et au sens commun »(BO, 2013, p. 2).

Les réflexions épistémologiques et didactiques menées en formation avec les enseignants débutants semblent déboucher sur leur adhésion à ce principe épistémologique. Pour autant, ils rencontrent souvent des difficultés importantes dans la mise en œuvre, dans leurs classes, de démarches didactiques s'appuyant sur cette conception épistémologique de la discipline.

Cet article se veut alors un des éléments ${ }^{1}$ d'une " carte légendée ", permettant, selon Jean-Pierre Astolfi, de faire « le tour d'horizon essentiel des possibles et des impossibles » (Astolfi, 1993, p. 9) dans le cadre d'une "recherche de faisabilité ", d'abord praxéologique : il s'agit d'établir un corpus, sur la base d'études de cas, des difficultés rencontrées par les professeurs débutants dans la mise en œuvre de la démarche didactique choisie. Ces difficultés sont ensuite travaillées en formation. L'expérimentation analysée ici concerne la définition du concept de population active, prélude à une étude du phénomène du chômage, en classe de Seconde. 


\section{ÉPISTÉMOLOGIE ET DIDACTIQUE DES SCIENCES ÉCONOMIQUES ET SOCIALES}

Une conception épistémologique de la discipline qui s'oppose à l'inductivisme et aux «fondamentaux»

La réflexion menée depuis une dizaine d'années maintenant en didactique des SES cherche à rompre, tant avec une certaine épistémologie de la discipline scolaire, longtemps centrée autour de la démarche inductive, qu'avec une autre approche plus récente émanant de certains membres du corps de l'Inspection, et tendant à promouvoir un recentrage sur les «fondamentaux», définis comme ce qui, dans une des disciplines de référence des SES (l'économie ou la sociologie) fait l'objet d'un consensus entre les universitaires.

\section{Un projet fondateur inductiviste}

Lorsque la discipline scolaire sciences économiques et sociales (SES) se crée en 1966-67, les textes originaux définissent ce que certains nommeront plus tard le « projet fondateur ${ }^{2}$ », qui présente trois caractéristiques majeures (Dollo, 2005b, p. 85) : - il s'agit d'une part de conduire les élèves à l'intelligence des économies et des sociétés contemporaines, c'est-à-dire à l'étude d'un certain nombre de questions, jusque-là absentes de l'enseignement secondaire général ; - il s'inscrit d'autre part dans une perspective de l'unité des sciences sociales, en soulignant que le découpage en disciplines étanches ne permet pas de rendre compte du social dans sa complexité. Il s'agit alors de partir de l'étude d'un certain nombre d' " objets » (la famille, la monnaie, le chômage) et de mobiliser de façon intégrée l'apport des diverses sciences sociales, en construisant une discipline scolaire « ad hoc » : "À la question "quelle économie enseigner dans le second cycle du second degré de l'enseignement de l'enseignement général ?" Marcel Roncayolo ${ }^{3}$ répond en "fabricant" une discipline véritablement nouvelle en ce sens qu'elle ne constitue pas un simple "modèle réduit" de ce qui se fait dans l'enseignement supérieur » (Brémond \& Lanta, 1995, p. 64) ;

- enfin, ce projet intègre une approche pédagogique novatrice qui emprunte ses princi- paux éléments aux divers courants pédagogiques qui critiquent l'école traditionnelle, la mémorisation mécanique, le cours magistral. Dans cette nouvelle discipline scolaire, on insiste sur la volonté de favoriser l'activité des élèves, le travail sur documents, la pratique des enquêtes.

Les textes officiels initiaux prônent alors une " pédagogie active » devant permettre à l'élève de « participer à l'élaboration de son propre savoir ». On met l'accent sur «l'observation directe », le « concret », la « description ». On encourage ainsi « la pratique d'une pédagogie active reposant pour une bonne part sur l'emploi de méthodes inductives » (instructions officielles de 1982).

Les théories ne sont pas évoquées, tout au plus les «mécanismes » que l'on propose de n'étudier que « progressivement, à partir d'exemples concrets». On préconise de mettre les élèves en activité mais on ne fait aucune référence à leurs conceptions ou représentations sociales du monde qui les entoure. On applique plutôt un « inductivisme naif » au sens de Alan Chalmers (1987, p. 20).

Ce projet fondateur mêlait ainsi étroitement une finalité (se confronter aux questions de société), des options épistémologiques (la possibilité de bâtir une discipline scolaire «ad hoc ») et un positionnement pédagogique fort (inductiviste).

Une approche par les "fondamentaux" excluant les débats théoriques de l'enseignement de la discipline

Plus récemment, une autre approche, émanant notamment de certains membres de l'Inspection, tend à promouvoir un enseignement de la discipline scolaire présentant une certaine progressivité : «L'apprentissage de toute discipline doit être progressif. Il me semble nécessaire de commencer par ce qui est à la fois fondamental et simple pour progressivement complexifier les choses et étudier les détails. Il est donc utile de pouvoir hiérarchiser les contenus »(Montoussé, 2004, p. 69).

Dans cette perspective, les professeurs de SES doivent pratiquer un certain recentrage sur les «fondamentaux »: « On peut définir le fondamental comme étant la base faisant l'objet d'un consensus entre les économistes ; l'étude des désaccords et donc du débat théorique relève alors d'un niveau supérieur 
de complexité et ne peut être envisagée qu'après que les élèves aient assimilé les concepts et mécanismes fondamentaux $[\ldots]$. L'épistémologie relève d'un niveau encore plus élevé. S'il est utile, voire nécessaire, que les professeurs de SES connaissent les différents problèmes épistémologiques de la science économique, il est préférable qu'ils ne l'enseignent pas à leurs élèves » (Montoussé, 2004, p. 69).

Selon cette conception de l'enseignement des sciences économiques et sociales, l'étude des débats théoriques ne doit donc pas être envisagée dans un premier temps, car d'un niveau trop complexe pour les élèves qui doivent d'abord, on l'aura compris, assimiler les concepts et mécanismes dits « fondamentaux ».

On est donc en présence, d'une part, d'une conception inductiviste à visée « critique » héritée $\mathrm{du}$ « projet fondateur » des SES et, d'autre part, d'une conception qui conduit à éliminer les interrogations théoriques et le débat scientifique dans la classe au profit d'une transmission des « fondamentaux ».

La conception défendue ici, au contraire, est fondée sur la nécessité d'initier les élèves à la méthode scientifique des disciplines de référence et repose sur des démarches d'apprentissage destinées à amener les élèves « à se poser des questions précises et à formuler, sur cette base, des hypothèses visant à résoudre le problème identifié » ( $\mathrm{BO} \mathrm{n}^{\mathrm{O}} 21$ du 23 mai 2013, p. 35) ainsi que l'indiquent d'ailleurs les nouveaux textes officiels.

Une épistémologie de la discipline fondée sur la rupture avec le sens commun

\section{Les disciplines de référence des sciences économiques et sociales sont des sciences}

À la suite notamment de Pierre Bourdieu (1992), notre hypothèse est en effet que "les sciences sociales sont soumises aux règles qui valent pour les autres sciences : il s'agit de produire des systèmes explicatifs cohérents, des hypothèses ou des propositions organisées en modèles parcimonieux capables de rendre compte d'un vaste nombre de faits observables empiriquement et susceptibles d'être réfutés par des modèles plus puissants, obéissant aux mêmes conditions de cohérence logique, de systématicité et de réfutabilité empirique [...]. Une journée typique de sociologue, avec ses tâtonnements expérimentaux, ses analyses statistiques, ses lectures d'articles spécialisés et ses discussions avec des collègues, ressemble tout à fait à celle d'un savant ordinaire » (Bourdieu, 1992, p. 159). Il n'y a donc pas, dans cette perspective, de dualisme épistémologique qui conduirait à considérer que les sciences sociales relèvent de normes épistémologiques radicalement différentes de celles qui valent pour les sciences de la nature.

Ainsi, le concept de rupture avec le sens commun, emprunté notamment à l'épistémologie de Gaston Bachelard (1938/1983), s'applique aux sciences sociales. La connaissance scientifique se construit donc contre l'évidence.

Pierre Bourdieu rappelle cette exigence de construction de l'objet scientifique : « construire un objet scientifique, c'est, d'abord et avant tout, rompre avec le sens commun, c'est-à-dire avec des représentations partagées par tous, qu'il s'agisse des simples lieux communs de l'existence ordinaire ou des représentations officielles, souvent inscrites dans des institutions, donc à la fois dans l'objectivité des représentations sociales et dans les cerveaux. Le préconstruit est partout » (Bourdieu, 1992, p. 207).

Cette approche épistémologique est donc en rupture avec la conception empiriste selon laquelle il faudrait « partir des faits ».

On considère au contraire que ce sont les concepts qui sont premiers, car eux seuls permettent de découper le réel en objets d'investigation et seuls ils permettent de poser des questions pertinentes susceptibles de guider à la fois l'investigation du chercheur et la structuration des connaissances produites par la recherche. Comme le soulignait Michel Fichant (1969), « une science ne naît pas de la définition d'un objet, ni de la rencontre d'un objet, ni de l'imposition d'une méthode. Elle naît de la constitution d'un corps de concepts, avec leurs règles de production. De ce fait, le développement d'une science c'est la formation des concepts et des théories de cette science » (p. 100).

\section{Problématisation et investigation scientifique}

Cette conception épistémologique de la discipline conduit à penser que la notion clé est celle de problème. Celui-ci apparait alors en effet comme le moteur de la progression scientifique : "La "logique de la découverte" consiste alors en ceci d'avoir à délimiter strictement le problème, de présenter des 
hypothèses les plus réfutables possible sur le plan empirique » (Johsua \& Dupin, 2003, p. 60). Les instructions officielles des derniers programmes scolaires de SES, indiquent, en 2013, qu'il faut amener les élèves « à se poser des questions précises et à formuler, sur cette base, des hypothèses visant à résoudre le problème identifié ».

Christian Orange (2003a), précise notamment qu'accéder à un savoir scientifique, ce n'est pas uniquement accéder à une " bonne » solution au regard des savoirs savants. C'est également passer d'une opinion à un savoir problématisé. Pour lui en effet, " le lien entre savoirs scientifiques et problématisation est fort, ceux-ci ne pouvant pas être simplement considérés comme des solutions à des problèmes, mais comme la trace même de la problématisation »(Orange, 2008, p. 39).

Dans cette perspective, il semble important de mettre en place dans la classe une logique d'investigation - structuration qui permette de mettre l'élève dans une posture de recherche, sur la base de la formulation d'un problème et d'hypothèses susceptibles de le résoudre. Le professeur est le concepteur d'une situation didactique qui vise à simuler les conditions du débat scientifique. Il s'agit de faire pratiquer par les élèves les règles du champ scientifique telles qu'elles sont décrites par Pierre Bourdieu (2001). Cette posture est alors de nature à montrer le rôle essentiel des controverses scientifiques dans la construction de la vérité.

Nous avons pu montrer (Dollo, 2001, 2002 ; Beitone, Dollo, Hemdane, \& Lambert, 2013), que les représentations sociales des élèves, ces connaissances «spontanées » ou "naturelles », forment le plus souvent une véritable construction opérée par l'élève en interaction active avec son environnement : les connaissances ne s'empilent pas les unes sur les autres, mais sont structurées. Certes, cet ensemble de connaissances est le plus souvent éloigné voire opposé à la connaissance scientifique. Mais pourtant, ces modèles construits par les élèves semblent efficaces et pertinents au regard de la vie courante. C'est d'ailleurs cela qui en fait parfois de véritables obstacles à l'apprentissage. Dès lors, tout dispositif d'apprentissage qui ne prend pas en compte les représentations peut manquer son objectif. Les apprentissages scolaires risquent d'acquérir (et de façon souvent éphémère) un caractère de savoir décoratif. L'élève sait quelle est la bonne réponse à fournir pour satisfaire le maître, mais ses représentations ne sont pas modifiées pour autant.
Cependant, la simple «prise en compte » des conceptions des élèves ne suffit pas. On peut en effet traiter les conceptions de manière "inductiviste ", comme si, par exemple, la présentation d'un " fait » contraire pouvait suffire à induire la nécessité d'un « changement conceptuel».

Une amélioration de cette attitude pourrait être de tenir compte de l'existence de plusieurs conceptions parmi les élèves, de les faire se confronter entre elles, de manière à favoriser l'émergence d'un conflit socio-cognitif. Mais il semble difficile de produire un tel changement au cours de conflits brefs (parfois une seule séquence d'une heure). Il est alors nécessaire de penser des dispositifs didactiques permettant non seulement l'émergence de conflits socio-cognitifs, mais aussi la formulation et la réfutation d'hypothèses par les élèves eux-mêmes.

On ne fait pas construire "naturellement " un modèle « correct » de compréhension de la réalité sociale. Le professeur doit alors élaborer un dispositif permettant de conduire à plusieurs hypothèses explicatives contradictoires, dont certaines seront éliminées en utilisant le débat entre pairs, par des arguments « logiques» (et qui vont être considérés comme tels par les élèves). Pour trancher entre celles qui subsistent, le professeur pourra avoir recours à des connaissances nouvelles (par le biais de textes scientifiques notamment).

Le « débat » proposé par le professeur n'est donc en rien naturel. Il ne peut être que la conséquence d'une construction artificielle, minutieuse et contraignante qui crée un cadre où la discussion apparaît - fictivement - comme libérée. En outre, le professeur dispose dans la discussion d'une autorité différente de celle des élèves (asymétrie des positions). Cependant, la mise en place d'un tel dispositif dans la classe crée une aire de «liberté » qui favorise une interaction sociale apte à aider l'apprentissage.

Dans la mise en ceuvre d'une logique d'investigation-structuration dans la classe, et pour reprendre une expression de Christian Orange, « ce qui se joue n'est pas simplement une aide au changement de conceptions, mais il s'y construit une part fondamentale des savoirs scientifiques visés » (Orange, 2003b).

Ainsi, le "débat scientifique dans la classe ", même s'il peut être mis en œuvre dans le cadre de situations de classes dites ordinaires, ne peut s'improviser. Il nécessite une bonne maîtrise scientifique du thème étudié, une connaissance a priori des conceptions des élèves et une programmation minu- 
tieuse. Par ailleurs, l'enseignant joue un rôle essentiel dans la conduite du débat.

La deuxième partie de ce texte propose l'analyse didactique d'une séance mise en œuvre par une professeure stagiaire. Celle-ci, dans le cadre d'une formation d'enseignants s'efforçant de dépasser les manières « ordinaires » de faire, s'essaie à renverser la démarche transmissive classique de la définition d'un concept catégoriel, celui de population active. Il s'agit alors d'analyser les difficultés que pose aux enseignants débutants la mise en œuvre d'une démarche conforme aux positions épistémologiques défendues plus haut.

\section{ANALYSE DIDACTIQUE D'UNE EXPÉRIMENTATION EN CLASSE DE SECONDE : DÉFINITION DE LA POPULATION ACTIVE À PARTIR D'UNE « DÉMARCHE D'INVESTIGATION "}

\section{Cadre théorique de l'analyse : la situation didactique comme jeu}

Nous ne présentons dans ce qui suit que les éléments de théorisation qui nous semblent pertinents pour l'analyse.

Née dans les années 1980 (Schubauer-Leoni, 1986), la théorie de l'action conjointe en didactique part du postulat central selon lequel « les phénomènes transpositifs sont le résultat d'une co-activité entre professeur et élèves relativement à des enjeux de savoirs » (Amade-Escot, 2013, p. 83). Ses développements (Schubauer-Leoni \& Leutenegger, 2007 ; Sensevy \& Mercier, 2007), s'appuient sur des concepts issus de théorisations antérieures, comme la transposition didactique (Chevallard, 1985) ou les situations didactiques (Brousseau, 1998).

Nous pensons, avec Yves Chevallard, que l'enseignant « a pour tâche [...] la prise en charge de la création des conditions de possibilité de l'apprentissage » (Chevallard, 1986, p. 8). Pour accomplir cette tâche, le professeur définit une situation qui l'implique " dans un jeu avec le système des interactions de l'élève avec les problèmes qu'il lui pose. Ce jeu ou cette situation est la situation didactique » (Brousseau, 1998, p. 60). Dans ce jeu coopèrent deux joueurs que sont l'Elève, joueur A (la majuscule indiquant qu'il s'agit ici d'une instance, il peut donc y avoir plusieurs joueurs, plusieurs élèves en l'occurrence) et le professeur, joueur B, en interaction autour d'un objet de savoir. Pour jouer, il faut connaitre le jeu et le professeur doit donc en définir les règles « constitutives » ou « définitoires» (Sensevy, 2007). Le caractère « conjoint » de l'action qui se mène dans ce jeu didactique est lié au fait que le professeur ne gagne que si l'autre joueur, l'Élève, gagne aussi, c'està-dire apprend : " c'est un jeu de savoir » (Sensevy, 2007 , p. 20). Pour cela, le professeur, après avoir défini le jeu, doit organiser les conditions de sa dévolution, définie par Guy Brousseau comme « l'acte par lequel l'enseignant fait accepter à l'élève la responsabilité d'une situation d'apprentissage (adidactique) ou d'un problème » (Brousseau, 1998 p. 303). La définition d'une situation doit donc aussi comprendre des « règles stratégiques qui font l'espace d'incertitude des élèves et définissent le problème qui leur sera peut-être dévolu » (Assude \& Mercier, 2007, p. 168). Ces règles définies, le professeur ne doit rien dévoiler « qui puisse amener A (l'Élève) à produire des stratégies gagnantes de faible valeur, parce que "pauvres en savoir " » (Sensevy, 2007, p. 20). L'élève doit « agir en première personne » et il doit donc assumer, à un certain moment, une forme de solitude dans l'apprentissage.

Définir et dévoluer constituent ainsi classiquement deux descripteurs permettant de rendre compte de l'action du professeur. Il faut leur en ajouter deux autres pour caractériser le jeu didactique : la régulation et l'institutionnalisation. Le professeur doit en effet « réguler, pendant la durée du jeu, les comportements des élèves en vue de la production, par ceux-ci, de stratégies gagnantes » (Sensevy, 2007, p. 28), sans pour autant se substituer à eux dans cette production. Mais ce jeu didactique n'a, au final, de sens « que dans les apprentissages qu'il permet à la fois de produire et de reconnaitre chez les élèves. [...] C'est le processus d'institutionnalisation (Brousseau, 1998), par lequel le professeur assure les élèves que leur activité leur a permis de retrouver des savoirs légitimes hors de l'institution-classe * (Sensevy, 2007, p. 29).

\section{Analyse préalable des savoirs en jeu dans la séance analysée}

\section{Le concept de population active en économie}

Si le concept de population active, en tant que tel, ne fait plus aujourd'hui partie de la colonne des 
« notions à découvrir » du programme de l'enseignement d'exploration de la classe de Seconde, il est cependant largement travaillé par les professeurs de SES avec leurs élèves. En effet, pour aborder la question du chômage ${ }^{3}$, les enseignants sont amenés à définir la population active puisque la définition d'un chômeur fait référence à l'activité au sens économique du terme.

La définition de ce concept catégoriel ne fait pas l'objet de controverses au sein de la communauté des économistes et sa définition est simple. Sur le site de l'INSEE, elle est formulée ainsi : « La population active regroupe la population active occupée (appelée aussi "population active ayant un emploi") et les chômeurs ».

La population active occupée, selon les recommandations du Bureau international du travail (BIT) adoptées depuis 1982, comprend les personnes (âgées de 15 ans ou plus) ayant occupé un emploi (ne serait-ce qu'une heure) au cours d'une semaine donnée (appelée semaine de référence), qu'elles soient salariées, à leur compte, employeurs ou aides dans l'entreprise ou l'exploitation familiale. Elle comprend aussi les personnes pourvues d'un emploi mais qui en sont temporairement absentes pour un motif tel qu'une maladie (moins d'un an), des congés payés, un congé de maternité, un conflit du travail, une formation, une intempérie, etc. Les militaires du contingent, les apprentis et les stagiaires rémunérés font partie de la population active occupée.

Toujours selon l'INSEE, un chômeur est une personne qui n'a pas d'emploi et qui en recherche un. La définition des chômeurs est extrêmement sensible aux critères retenus. La définition la plus couramment utilisée est celle du chômeur « au sens du BIT ». Elle permet d'effectuer des comparaisons internationales. Pour le BIT, un chômeur est une personne en âge de travailler (15 ans ou plus) qui répond simultanément à trois conditions :

- être sans emploi, c'est-à-dire ne pas avoir travaillé ne serait-ce qu'au moins une heure durant une semaine de référence ;

- être disponible pour prendre un emploi dans les 15 jours ;

- avoir cherché activement un emploi dans le mois précédent ou en avoir trouvé un qui commence dans moins de trois mois.

Ainsi, si le concept de population active est simple à définir, il renvoie cependant à deux autres concepts clés (et largement présents dans les programmes de sciences économiques et sociales au lycée), celui de travail au sens économique et celui d'emploi.

La notion d'emploi dans les conventions du BIT, est très large, puisqu'il suffit d'avoir travaillé pendant une durée quelconque, ne serait-ce qu'une heure, au cours d'une semaine dite de référence.

On le voit, cette définition du concept d'emploi renvoie à celui de travail. Or les difficultés conceptuelles et didactiques sont ici plus importantes.

D’une manière générale, le travail peut se définir comme « une activité humaine conduisant à la production ou à l'entretien des biens et services * (Beitone, Cazorla \& Hemdane, 2016, p. 615). Dans la conception marxiste, le travail est, encore plus généralement, toute activité de transformation de la nature (et de la société), et François Vatin (2009) montre ainsi que chez Marx, le travail n'est pas réductible au cadre social de sa gestion dans la société capitaliste: «Le travail est d'abord un procès qui se passe entre l'homme et la nature, un procès dans lequel l'homme règle et contrôle son métabolisme avec la nature par la médiation de sa propre action. Il se présente face à la matière naturelle comme une puissance naturelle lui-même. [...] Mais en agissant sur la nature extérieure et en la modifiant par ce mouvement, il modifie aussi sa propre nature » (Marx, 1867-1883, p. 199-200). Le travail est ainsi inhérent à l'espèce humaine. Pour autant, tout travail n'a pas forcément un enjeu économique.

Il faut ainsi distinguer en économie, le travail de l'emploi, ce dernier étant une des formes d'institutionnalisation du travail.

\section{Vocabulaire courant versus vocabulaire scientifique : source de difficultés didactiques}

D'une manière générale, il existe une difficulté didactique relative à l'articulation problématique, pour les élèves, entre le vocabulaire courant et le vocabulaire scientifique. Ainsi que le précisent Pierre Bourdieu, Jean-Claude Chamboredon et JeanClaude Passeron, « dans la mesure où le langage ordinaire et certains usages savants des mots ordinaires constituent le principal véhicule des représentations communes de la société, c'est sans doute une critique logique et lexicologique du langage commun qui apparaît comme le préalable le plus indispensable à l'élaboration contrôlée des notions scientifiques » (Bourdieu, Chamboredon \& Passeron, 1968, p. 28). 
Certaines difficultés langagières des élèves en SES tiennent ainsi au fait qu'ils ne comprennent pas toujours pourquoi on n'utilise pas les mêmes « mots » en SES et dans la vie courante : par exemple, en ce qui concerne l'emploi et le chômage, « les élèves se réfèrent de façon importante à une idée "commune" de la notion d'activité : est actif, quelqu'un qui "fait" quelque chose, qui agit, et notamment qui travaille. On parle ainsi de l'activité professionnelle. Or, le chômeur ne travaille pas, il n'a pas d'activité au sens courant de ce terme. Les élèves ont ainsi du mal à percevoir l'aspect conventionnel de la définition de certains concepts économiques » (Dollo \& Johsua, 2002, p. 192). Cette difficulté comme obstacle aux apprentissages se retrouve dans les propos de certains élèves interrogés par l'équipe d'Élisabeth Bautier et de Jean-Yves Rochex. C'est ainsi que ces auteurs notent que Séverine, « qui ne peut admettre qu'un ménage au sens statistique puisse être composé d'une seule personne ou qu'un chômeur puisse faire partie de la population active, n'est pas loin de penser que son professeur "en rajoute" et que tout irait mieux s'il expliquait "comme nous on parle" » (Bautier \& Rochex, 1998, p. 280). Ils citent également le cas de Valérie qui a bien retenu de ses cours de SES que les termes tels que ménage, actifs, etc. pouvaient y avoir une autre signification que dans le langage courant, mais c'est pour déplorer qu'il en soit ainsi : « ça devrait être employé comme ça dans la vie courante, mais on ne l'emploie pas... Je voudrais parler de façon à ce que, en cours et en dehors, ce soit la même chose, ce soient les mêmes mots, le même vocabulaire » (Bautier \& Rochex, 1998, p. 281).

\section{Enjeux de savoir, obstacles didactiques et démarches « ordinaires"}

L'enjeu de savoir est ici important, car il permet, au-delà de la simple question des contours de la population active, de mettre en évidence un point essentiel de la démarche de l'économiste : de nombreux concepts sont définis sur la base de conventions statistiques qui donnent à la notion un sens parfois très éloigné du langage courant. La plus élémentaire des données statistiques repose ainsi sur des définitions et des conventions dont il faut comprendre la logique. Un premier enjeu didactique se situe bien ici dans le passage du sens courant au sens scientifique. Le professeur doit donc engager les élèves dans un processus de secondarisation, pour reprendre l'expression d'Élisabeth Bautier et Roland Goigoux (2004). Les auteurs reprennent la distinction faite par Mikhaill Bakhtine (1984) entre genres de discours premiers et genres seconds : « les genres premiers peuvent ici être décrits comme relevant d'une production spontanée, immédiate, liée au contexte qui la suscite et n'existant que par lui, les ressaisissent dans une finalité dans l'oubli qu'un quelconque apprentissage ou travail sous-jacent. [...] Les genres sont seconds, lorsque, fondés sur les premiers, ils les travaillent, les ressaisissent dans une finalité qui évacue la conjoncturalité de leur production, ils supposent une production discursive qui signifie bien au-delà de l'interaction dans laquelle dans laquelle elle peut conjoncturellement se situer » (Bautier \& Goigoux, 2004, p. 91).

Un second enjeu didactique existe dans le passage du concept à la mesure. Quels sont les critères qui permettent d'identifier le fait qu'un individu est actif au sens économique, quand est-il classé « en emploi », quand est-il classé chômeur ? Ainsi, « le fait de définir les chômeurs comme des actifs repose sur l'idée selon laquelle la population active doit mesurer la quantité de travail disponible pour une économie à un moment donné. [...] L'effectif de la population active étant déterminé, une autre question se pose, celle de savoir quelle est la part de cette population active effectivement utilisée dans la production (actifs occupés) et quelle est la part qui ne l'est pas (chômeurs)» (Beitone, 2005, p. 24).

Élisabeth Bautier et Jean-Yves Rochex identifient par ailleurs des conduites enseignantes pouvant avoir des effets potentiellement contre-productifs du point de vue de l'apprentissage des élèves. Et notamment, certaines pratiques enseignantes pêcheraient «par sur-ajustement aux difficultés et aux caractéristiques des élèves : par souci de faciliter leur réussite et de préserver leur image de soi, on leur propose des tâches simplifiées à l'excès, morcelées et ne faisant appel qu'à des compétences cognitives de "bas niveau" et à des situations fermées, dans lesquelles l'activité des élèves est pour l'essentiel déterminée et contrôlée de l'extérieur, par les procédures et les consignes et/ou par l'enseignant $»$ (Bautier \& Rochex, 2004, p. 97-98). Cette position rejoint largement notre conception épistémologique des SES.

Pour autant, plusieurs manuels scolaires de la classe de Seconde proposent directement dans un document (un extrait de texte ou un schéma), une 
définition de la population active, livrée « brute» aux élèves. Aucune tâche réflexive n'est proposée. La définition du concept est donnée, les critères de classement également. Il est simplement demandé aux élèves de lire, voire, au mieux, d'appliquer cette définition en classant quelques exemples.

Les séquences d'enseignement sur la définition de la population active se déroulent très souvent de façon conforme à ce qui est proposé dans ces manuels scolaires : les enseignants donnent aux élèves (soit directement, soit par la lecture d'un bref texte ou schéma) la définition de la population active, puis leur demandent ensuite de résoudre un petit exercice destiné à vérifier leur compréhension de la notion. Il s'agit, le plus souvent, d'une liste d'individus qui doivent être classés comme actifs ou inactifs, au sens économique de l'activité.

Les élèves «apprennent " ainsi une définition de la population active et sont généralement tout à fait capables de la restituer lors d'un contrôle. Mais ils ne se posent pas véritablement de questions sur les raisons d'un tel classement. On se retrouve bien ici dans le cadre d'une absence de dévolution d'un problème. En outre, l'enjeu d'une introduction à la population active va bien au-delà de sa définition. Les conventions à l'œuvre influencent de façon sensible la façon d'appréhender le phénomène du chômage notamment. En fonction des conventions à l'œuvre en effet, les indicateurs de mesure du chômage varient notablement. Or ce sont ces enjeux-là qu'il convient de faire découvrir aux élèves à travers le fait que les chômeurs font partie de la population active.

\section{Définition de la population active à partir d'une démarche « d'investigation »}

\section{Les objectifs et la démarche de l'enseignante}

Charlotte, la professeure stagiaire, dont un extrait de séance est analysé ici souhaitait expérimenter un des « scénarios » travaillés en formation, en l'adaptant au thème du chômage et à la définition de la population active.

La séance de cours filmée inaugure un nouveau chapitre de la classe de Seconde. Charlotte choisit de renverser le « schéma classique » proposé notamment par certains manuels scolaires. Son objectif est alors de faire construire la définition de la population active par les élèves.
La séquence débute par une remobilisation des connaissances acquises lors de chapitres précédents. Charlotte demande notamment aux élèves de rappeler la définition de la science économique, donnée dans l'introduction générale : «science qui étudie la production, la répartition et la distribution des richesses ». Puis, par un jeu de questions / réponses, elle introduit l'idée selon laquelle les économistes s'intéressent à la production de richesses, et que, pour produire, on fait appel à des facteurs de production, notamment le capital et le travail. Elle demande également aux élèves de rappeler la définition du travail au sens économique du terme (étudiée dans le chapitre sur la production). Ainsi, le travail renvoie à l'emploi, et pour étudier l'emploi, les économistes utilisent notamment le concept de population active.

Le jeu est alors présenté aux élèves :

«Donc nous, on va chercher la définition économique, donc la définition qui est donnée par la science économique, de la population active. Alors, pour ça on va faire l'activité 1 que vous avez dans votre polycop. On fait le tableau, là. Donc vous prenez le tableau et on va le faire ensemble. Alors, on va essayer de classer... C'est Bon? Donc les différents exemples entre actifs et inactifs. »

La première tâche proposée aux élèves consiste ainsi en un tableau à trois colonnes présentant, en ligne, différents individus. Les règles « constitutives » du jeu (Sensevy, 2007) sont clairement énoncées : il s'agit de classer chaque individu dans une des catégories proposées (actif ou inactif), les élèves ne connaissant pas encore la définition économique de la notion de population active.

Pour chaque exemple, traité oralement, l'enseignante initie une discussion entre les élèves lorsqu'il y a désaccord sur le classement à opérer. Elle laisse la parole à différents élèves en leur demandant d'argumenter leur réponse. Les « cas à problème » sont notés au tableau et elle indique aux élèves qu'on y reviendra plus tard. Après chaque échange sur l'un des cas discutés, elle donne la « réponse » de l'économiste.

La seconde tâche doit permettre aux élèves, en fonction du tableau rempli précédemment, de déterminer les critères à l'origine du classement, par les statisticiens et les économistes, d'un individu dans la catégorie actif ou inactif. 


\section{Déroulement du jeu et questionnement des élèves}

Amélie est interrogée sur le premier exemple qui ne pose aucune difficulté aux élèves (voir tableau 1).

Tableau 1. L'exemple de l'ouvrier

\begin{tabular}{|l|l|}
\hline Amélie & $\begin{array}{l}\text { Un ouvrier dans une usine Ford, c'est un } \\
\text { actif }\end{array}$ \\
\hline P. & $\begin{array}{l}\text { Alors est-ce que tout le monde est } \\
\text { d'accord avec ça? }\end{array}$ \\
\hline É. & Oui \\
\hline P. & $\begin{array}{l}\text { Oui, c'est bon ? Alors l'économiste il est } \\
\text { d'accord, c'est un actif. Le deuxième. } \\
\text { Manon? }\end{array}$ \\
\hline
\end{tabular}

Le choix de ce premier exemple permet à l'enseignante de démarrer le jeu, de dévoluer le problème aux élèves et de les enrôler dans la tâche. Il n'y a ici aucun «danger » dans la mesure où il apparaît évident à tous les élèves qu'un salarié dans une entreprise est un actif au sens économique. Ils comprennent ainsi rapidement la situation mise en place : un élève est interrogé, s'il n'y a pas de discussion sur l'exemple, l'enseignante valide la réponse, en l'occurrence celle de l'économiste comme elle le précise très clairement ( l'économiste, il est d'accord »). Les élèves cochent la « bonne case » dans leur tableau.

Le deuxième exemple doit permettre d'engager un échange d'arguments entre les élèves: tous ne sont pas d'accord sur le classement à opérer.

Tableau 2.

L'exemple de la femme et de l'homme au foyer

\begin{tabular}{|l|l|}
\hline Manon & $\begin{array}{l}\text { Une femme ou un homme au foyer, } \\
\text { c'est inactif }\end{array}$ \\
\hline P. & $\begin{array}{l}\text { Alors, inactif, est-ce que tout le } \\
\text { monde est d'accord avec ça? }\end{array}$ \\
\hline É. & Oui... \\
\hline É. & Non... \\
\hline P. & $\begin{array}{l}\text { Alors, qui n'est pas d'accord ? Anne, } \\
\text { pourquoi ? }\end{array}$ \\
\hline Anne & $\begin{array}{l}\text { Ben ça dépend s'ils sont au chômage } \\
\text { ou non? }\end{array}$ \\
\hline Marine & Au sens économique c'est inactif \\
\hline P. & $\begin{array}{l}\text { Alors, au sens économique, tu nous } \\
\text { dis, c'est inactif. Anne elle nous dit } \\
\text { que c'est actif. Pourquoi, Anne, ça } \\
\text { serait classé dans les actifs? }\end{array}$ \\
\hline
\end{tabular}

\begin{tabular}{|l|l|}
\hline Anne & Il faut faire tourner la maison \\
\hline É. & $\begin{array}{l}\text { Alors ça voudrait dire que l'homme } \\
\text { au foyer, ou la femme, il est actif, il } \\
\text { travaille, il fait tourner la maison. } \\
\text { Alors comment on a défini le travail ? }\end{array}$ \\
\hline Anne & Ah, rémunéré... \\
\hline P. & $\begin{array}{l}\text { Alors là, bon on se pose la question, } \\
\text { on le marque d'accord. Donc homme } \\
\text { ou femme au foyer on a un petit } \\
\text { souci. Pourquoi tu nous dis qu'il est } \\
\text { inactif Marine? }\end{array}$ \\
\hline Marine & $\begin{array}{l}\text { Parce que, enfin il est actif parce que } \\
\text { bien sûr il fait quelque chose à la } \\
\text { maison, mais aux yeux de la société, } \\
\text { il fait rien. Enfin pour la société il fait } \\
\text { rien. }\end{array}$ \\
\hline P. & $\begin{array}{l}\text { Tout le monde est d'accord avec ça? } \\
\text { Caroline? }\end{array}$ \\
\hline Caroline & $\begin{array}{l}\text { Un homme ou une femme au foyer } \\
\text { ne touche pas de rémunération, donc } \\
\text { il est pas vraiment actif. }\end{array}$ \\
\hline P. & $\begin{array}{l}\text { Oui ? Donc, on n'a pas de travail au } \\
\text { sens d'activité rémunérée. OK. Donc } \\
\text { l'économiste, il est d'accord, il le } \\
\text { classe bien dans les inactifs. }\end{array}$ \\
\hline
\end{tabular}

Cet exemple de l'homme ou de la femme au foyer, permet de faire émerger une conception de l'activité au sens courant (on est actif lorsqu'on " fait quelque chose ») qui va être discutée. Lors de la régulation de cette phase du jeu, Charlotte intervient cependant très tôt, à partir de la simple proposition d'une élève, " il faut faire tourner la maison », en introduisant la notion de travail au sens économique du terme («Alors, comment on a défini le travail ?»), pas encore formulée par les élèves. Ce rappel amène immédiatement les élèves à faire le lien entre travail au sens économique et rémunération, ce qui clôture très rapidement le débat sur ce « cas »: « donc, on n'a pas de travail au sens d'activité rémunéré [...]». Charlotte, ici, ne pousse pas au bout la logique de la réticence didactique au sens de Gérard Sensevy (2007). Elle dévoile très vite un élément de savoir et oriente les élèves sur un critère de classification des individus dans la population active. Mais, ce faisant, elle commet elle-même une erreur car ce critère n'est évidemment pas le seul : les chômeurs sont actifs et n'exercent pas de travail rémunéré. Pourtant, cet exemple aurait mérité un développement plus approfondi. Anne, en effet, dit : « ben ça dépend s'ils sont au chômage ou non ? ». Cette remarque pertinente de la part de cette élève n'est pas relevée par l'ensei- 
gnante et soulève le flou de l'exemple proposé par Charlotte : « un homme ou une femme au foyer » est une formulation trop générale. En effet, le fait d'être « au foyer » peut recouvrir plusieurs situations.

Il aurait ainsi fallu distinguer plusieurs cas. Celui, par exemple de monsieur Dupont qui a pris un congé parental pour s'occuper de ses enfants et de la maison pendant que son épouse occupe un poste important dans une grande entreprise. Il travaille, il transforme la nature (la maison était sale, elle devient propre), mais ce travail, dans l'état actuel des choses, ne correspond pas à un emploi. Monsieur Dupont est inactif ici. Le jour où il arrivera en fin de congé parental et retrouvera son emploi, la famille sera peut-être amenée à employer une assistante maternelle ou une aide ménagère. Le travail auparavant effectué par monsieur Dupont correspondra alors luimême à un emploi. Ce n'est donc pas le contenu de la tâche qui détermine l'enjeu économique du travail, mais son caractère institutionnalisé.

À côté de monsieur Dupont, on pourrait également évoquer monsieur Dupuis, dont l'entreprise vient de fermer et qui a donc été licencié pour motif économique. Il reste à son domicile pour effectuer une recherche d'emploi et utilise son temps libre pour prendre en charge le travail à effectuer dans la maison et auprès des enfants. Monsieur Dupuis est chômeur, et donc actif. Les exemples du retraité et du chômeur sont ceux qui posent le plus de problèmes aux élèves.

Tableau 3.

L'exemple du retraité

\begin{tabular}{|l|l|}
\hline P. & Alors, le suivant. Alexandra ? \\
\hline Alexandra & Un retraité c'est inactif \\
\hline Amélie & Ben Non ! \\
\hline É. & Ben si \\
\hline P. & $\begin{array}{l}\text { Alors, Alexandra, tu nous expliques } \\
\text { pourquoi tu le classes dans les inac- } \\
\text { tifs ? }\end{array}$ \\
\hline Alexandra & $\begin{array}{l}\text { Ben, parce que il est rémunéré, on lui } \\
\text { donne de... enfin... On lui donne } \\
\text { de l'argent, mais en même temps, il } \\
\text { travaille pas, il travaille plus. }\end{array}$ \\
\hline P. & Donc il n'a pas ? \\
\hline Alexandra & Hummm... D'emploi ? \\
\hline P. & $\begin{array}{l}\text { D'emploi. Donc toi tu le classerais en } \\
\text { tant qu'inactif ? }\end{array}$ \\
\hline Alexandra & Oui \\
\hline
\end{tabular}

\begin{tabular}{|l|l|}
\hline P. & Bon, OK. Qui a dit non ? Amélie ? \\
\hline Amélie & $\begin{array}{l}\text { Ben moi je le classerais comme } \\
\text { actif parce que il a eu un emploi et } \\
\text { c'est pour heu... Enfin c'est quand } \\
\text { même un peu la continuité même s'il } \\
\text { travaille plus quoi. Je veux dire, il est } \\
\text { rémunéré toujours par rapport à cet } \\
\text { emploi qu'il avait avant. }\end{array}$ \\
\hline P. & $\begin{array}{l}\text { En fait, le problème c'est que... } \\
\text { Qu'on a une séparation entre le } \\
\text { travail et la rémunération. Il est } \\
\text { rémunéré mais il a plus d'emploi. } \\
\text { Oui, c'est donc ça qui vous pose } \\
\text { problème? }\end{array}$ \\
\hline Amélie & $\begin{array}{l}\text { C'est par rapport à ce qu'il a fait } \\
\text { avant. }\end{array}$ \\
\hline P. & $\begin{array}{l}\text { C'est par rapport à ce qu'il a fait } \\
\text { avant... Alors on va le mettre là, on } \\
\text { a un problème avec le retraité ; alors } \\
\text { l'économiste, il le classe comme inac- } \\
\text { tif. Alors l'exemple suivant. }\end{array}$ \\
\hline
\end{tabular}

L'exemple du retraité conduit l'enseignante à se retrouver en quelque sorte " piégée » par la piste lancée plus tôt. Les élèves gardent en mémoire l'idée évoquée plus haut selon laquelle une activité au sens économique est une activité rémunérée. En revanche, ils ne font pas la distinction entre la pension de retraite et les revenus des salariés. Certains élèves cherchent les arguments qui permettraient de dire que le retraité est une personne active puisqu'elle est rémunérée. Amélie souligne ainsi le fait qu'« il est rémunéré toujours par rapport à cet emploi qu'il avait avant ». Et, sur ce point elle a raison. Mais le retraité ne perçoit plus un revenu d'activité et Charlotte, là encore, clôture rapidement le débat. On le voit, la question de la "rémunération » est ici un problème pour l'enseignante qui, alors qu'elle était intervenue trop précocement lors du cas de «l'homme ou la femme au foyer » (le travail au sens économique est rémunéré), ne rebondit pas ici sur le vocabulaire: un retraité ne perçoit en effet pas de salaire mais une pension de retraite. Or, la rémunération qu'est la pension de retraite n'est plus versée en contrepartie du fait qu'on occupe un emploi. Il s'agit d'un revenu assurantiel qui est un droit acquis parce que l'on a occupé un emploi dans le passé et versé à ce titre des cotisations.

Ici encore, Charlotte aurait pu proposer une variante dans les exemples. Car, jusqu'à un certain âge, un retraité peut, tout en gardant son statut de retraité, occuper un emploi. Il sera alors considéré comme actif. De la même façon, on aurait pu enrichir 
les possibilités en évoquant une personne vivant des revenus d'un capital, un « rentier » par conséquent. Là encore, ce n'est pas le revenu qui va permettre de classer cet individu, mais sa situation vis-à-vis de l'emploi. Un rentier sans emploi est inactif. La classification du chômeur est également source de difficultés chez les élèves.

Tableau 4. L'exemple du chômeur

\begin{tabular}{|c|c|}
\hline Marie & Un chômeur, ben il est... in.. actif? \\
\hline P. & Alors un chômeur il est actif? \\
\hline Marie & Inactif \\
\hline P. & $\begin{array}{l}\text { Alors, qui est d'accord, pas d'accord? } \\
\text { Alexandra? }\end{array}$ \\
\hline Alexandra & $\begin{array}{l}\text { Moi je suis pas d'accord parce que le } \\
\text { chômeur il est considéré... Enfin... } \\
\text { Heu... }\end{array}$ \\
\hline É. & Rires \\
\hline Alexandra & $\begin{array}{l}\text { Il est censé chercher du travail... } \\
\text { Donc... Ils lui donnent un peu d'in- } \\
\text { demnités et tout mais... Il est quand } \\
\text { même considéré comme actif }\end{array}$ \\
\hline P. & $\begin{array}{l}\text { Comme actif, ok. Donc on garde ton } \\
\text { argument. Alors, Lucas, tu voulais } \\
\text { intervenir tout à l'heure? }\end{array}$ \\
\hline Lucas & $\begin{array}{l}\text { Ouai, moi je disais aussi actif,... } \\
\text { Heu inactif parce que il fait rien quoi } \\
\text { pou... heu... pour augmenter le PIB } \\
\text { annuel de la France? }\end{array}$ \\
\hline P. & Alors, il fait rien. Alors, Anne? \\
\hline Anne & $\begin{array}{l}\text { Mais il touche de l'argent, l'État lui } \\
\text { donne de l'argent. }\end{array}$ \\
\hline Amélie & $\begin{array}{l}\text { Oui mais le retraité aussi à ce } \\
\text { moment-là ! }\end{array}$ \\
\hline P. & Parce qu'il a déjà travaillé ? \\
\hline Anne & $\begin{array}{l}\text { Oui, il faut avoir travaillé pour être } \\
\text { chômeur }\end{array}$ \\
\hline P. & $\begin{array}{l}\text { Alors dans ce cas-là on va... Donc... } \\
\text { Adrien et Martin ! Donc tu le classes } \\
\text { parmi les actifs, les inactifs... Les } \\
\text { actifs ou les inactifs? }\end{array}$ \\
\hline Anne & Parmi les actifs \\
\hline P. & $\begin{array}{l}\text { Parmi les actifs parce qu'il aurait une } \\
\text { rémunération? Qui serait une indem- } \\
\text { nisation en fait. }\end{array}$ \\
\hline Anne & Oui \\
\hline P. & $\begin{array}{l}\text { Est-ce que l'indemnisation elle est liée } \\
\text { à... enfin au fait que à un moment } \\
\text { donné il a eu un emploi ? Est-ce que } \\
\text { cette indemnisation elle est liée à } \\
\text { l'activité qu'il est en train de faire? }\end{array}$ \\
\hline
\end{tabular}

\begin{tabular}{|c|c|}
\hline Anne & Ben heu non... Heu les deux... \\
\hline P. & C'est-à-dire? \\
\hline Anne & $\begin{array}{l}\text { Ben il a... enfin... il est payé parce } \\
\text { qu'il a travaillé }\end{array}$ \\
\hline P. & Oui? \\
\hline Anne & $\begin{array}{l}\text { Mais il est payé parce qu'il ne travaille } \\
\text { plus aussi. Mais il a travaillé }\end{array}$ \\
\hline P. & $\begin{array}{l}\text { Alors est-ce que les indemnités } \\
\text { qu'il peut toucher, elles sont liées à } \\
\text { l'emploi qu'il occupe en ce moment? }\end{array}$ \\
\hline É. & Non \\
\hline P. & Non pourquoi? \\
\hline É. & Il est au chômage \\
\hline P. & $\begin{array}{l}\text { Parce qu'il est au chômage. Donc il } \\
\text { va falloir distinguer, quand on dit } \\
\text { travail, activité rémunérée, le fait que } \\
\text { l'individu il touche des indemnités } \\
\text { et tous les chômeurs ne touchent pas } \\
\text { des indemnités... }\end{array}$ \\
\hline É. & Ah bon ? \\
\hline P. & $\begin{array}{l}\text { Et... le fait qu'il est payé sur une acti- } \\
\text { vité. Donc le chômeur a priori c'est } \\
\text { pas sur ça qu'il est payé. Alors est-ce } \\
\text { que vous avez d'autres arguments? } \\
\text { Marine? }\end{array}$ \\
\hline Marine & $\begin{array}{l}\text { Moi le chômeur je le mettrais dans } \\
\text { actif parce que le retraité, moi je le } \\
\text { mets dans inactif parce qu'on sait } \\
\text { qu'il va pas retravailler tandis que } \\
\text { le chômeur il est à la recherche de } \\
\text { travail donc... Ça veut dire que forcé- } \\
\text { ment il va travailler. }\end{array}$ \\
\hline P. & $\begin{array}{l}\text { Forcément il va travailler. Donc ça } \\
\text { veut dire que... }\end{array}$ \\
\hline Lucas & Oui mais pas encore \\
\hline P. & $\begin{array}{l}\text { Alors Lucas? Écoutez la formulation } \\
\text { de Lucas }\end{array}$ \\
\hline Lucas & $\begin{array}{l}\text { Il est pas encore actif... Enfin, il est... } \\
\text { toujours inactif }\end{array}$ \\
\hline P. & $\begin{array}{l}\text { Alors en fait il est inactif mais il va } \\
\text { redevenir actif. }\end{array}$ \\
\hline Lucas & Voilà \\
\hline \multirow[t]{2}{*}{ P. } & $\begin{array}{l}\text { Bon alors on a quand même un pro- } \\
\text { blème avec notre chômeur. }\end{array}$ \\
\hline & {$[\ldots]$} \\
\hline P. & $\begin{array}{l}\text { Bon on va voir. On met les chômeurs } \\
\text { dans les situations à problème. Léco- } \\
\text { nomiste, il le classe en tant qu'actif. }\end{array}$ \\
\hline É. & Ah $! \ldots$ J'ai raison... \\
\hline
\end{tabular}


Là encore, le chômeur est associé à une rémunération. La professeure s'appuie cependant sur les propos d'Alexandra qui évoque, elle, des « indemnités ». L'enseignante rectifie ainsi le vocabulaire en parlant d'indemnisation du chômage et en essayant d'amener les élèves à réfléchir au fait que ces indemnisations ne sont pas liées à une activité aujourd'hui.

L'enseignante prend ici conscience du fait que la « rémunération » comme critère principal de classification des actifs s'impose chez les élèves. Cependant, le chômeur est bien un actif, mais ce statut n'est pas lié à la rémunération de son activité présente. Il est actif parce que présent sur le marché du travail, à la recherche d'un emploi. C'est bien une ressource en travail disponible pour l'économie à un moment donné selon la définition évoquée plus haut.

C'est une difficulté que l'enseignante n'avait pas anticipée. Elle ne relève d'ailleurs pas, à cet instant précis, le fait qu'Amélie avance l'idée que le retraité lui aussi, touche "de l'argent", pour un travail qu'il a eu.

À la fin de la discussion sur cet exemple, Marine introduit cependant la distinction entre le retraité et le chômeur sur la base du fait que l'un ne travaillera plus alors que l'autre devrait travailler à nouveau. On le voit ici, l'échange entre les élèves peut conduire à l'évocation d'arguments "justes», au sens économique. Et sur ce cas, Charlotte laisse davantage les échanges se dérouler, mais n'entend pas tous les arguments qu'elle pourrait saisir d'un point de vue didactique. Marine détient en effet « la clé » de la différence entre le retraité et le chômeur !

\section{Identification des critères utilisés par les économistes pour définir la population active}

À l'issue de cette tâche, les élèves se trouvent ainsi face à leur document rempli (les «bonnes » cases sont cochées) et à une liste d'exemples « à problème " inscrits au tableau. La tâche suivante est présentée par Charlotte:

« Alors. À partir de maintenant, vous savez comment l'économiste il a classé. Vous savez aussi que bon vous n'êtes pas d'accord, que il y en a qui classent... Bon par exemple un homme ou une femme au foyer, retraité ou chômeur, vous n'êtes pas d'accord sur la manière de classer actif, inactif. Donc vous voyez bien que, à un moment donné, il faut que l'économiste il donne une définition, qu'il construise une définition. Alors je vais vous demander, maintenant, à l'écrit, de rechercher les différents critères qu'a utilisés l'économiste pour définir ce qu'était un actif... Donc... Vous prenez 5 minutes à l'écrit pour définir les critères, à partir des exemples, qu'a choisi l'économiste pour définir ce qu'était un actif...»

Pendant la phase de réflexion sur les critères de classement entre actifs et inactifs, Amélie interpelle publiquement l'enseignante. Elle lui explique que les classements du retraité et du chômeur lui posent vraiment problème : ils ont tous les deux travaillé avant. Ils sont tous les deux rémunérés, tous les deux ne travaillent plus actuellement. Alors pourquoi l'un est-il actif et l'autre inactif?

Tableau 5.

Amélie a un problème

\begin{tabular}{|l|l|}
\hline P. & $\begin{array}{l}\text { Il y a un critère qui différencie les retrai- } \\
\text { tés et les chômeurs pour l'économiste. Il } \\
\text { faut le trouver }\end{array}$ \\
\hline Amélie & $\begin{array}{l}\text { Ah voilà. Comme le retraité il n'est pas à } \\
\text { la recherche d'un emploi parce qu'il a pris } \\
\text { sa retraite, alors il est considéré comme } \\
\text { inactif }\end{array}$ \\
\hline P. & Oui \\
\hline Amélie & D'accord ! \\
\hline
\end{tabular}

Le critère de privation d'emploi pour les chômeurs qui demeurent sur le marché du travail à la recherche du travail ou de cessation (volontaire ou par atteinte de la limite d'âge) d'une activité pour les retraités qui se retirent ainsi du marché du travail s'impose dans l'esprit des élèves.

Malgré les difficultés rencontrées par Charlotte, on voit ici que son effort de "renverser » le schéma classique consistant à proposer aux élèves une définition à illustrer, a un impact positif sur le questionnement des élèves. Dans le scénario "classique ", Amélie ne se serait sans doute pas (et l'ensemble de la classe non plus par conséquent) posé la question de la différence entre les statuts du chômeur et du retraité.

À l'issue de la réflexion individuelle, l'enseignante demande aux élèves de donner les critères retenus par l'économiste. Les élèves devaient trouver qu'une activité au sens économique du terme remplit plusieurs conditions : elle est rémunérée, elle est légale (le trafiquant de drogue, qui faisait partie des exemples à classer dans la tâche précédente, n'est donc pas actif), elle est déclarée ${ }^{4}$. Les indivi- 
dus seront considérés comme actifs s'ils exercent ou cherchent à exercer une telle activité.

Les exemples ayant entrainé une discussion sont les suivants: un homme ou une femme au foyer, un retraité, un chômeur, un journaliste qui travaille 20 heures par semaine, un trafiquant de drogue. Ces exemples sont repris sur la base des critères définis collectivement et l'enseignante s'assure que tous les élèves ont compris pourquoi ils sont classés dans telle ou telle catégorie. Le processus d'institutionnalisation (Brousseau, 1998 ; Sensevy, 2007) est ici bien mené par l'enseignante.

Pour autant, certaines difficultés récurrentes rencontrées par les professeurs débutants se font jour ici : crainte de la parole des élèves et, à certains moments, «fermeture » du jeu en orientant les élèves sur des « pistes» de réponses; crainte du manque de temps qui conduit l'enseignante à réduire le temps d'interaction sur certains exemples pourtant moteurs de l'avancée du savoir ; flou dans la proposition de certains exemples.

\section{CONCLUSION : UNE DÉMARCHE DIFFICILE À METTRE EN GUVRE POUR L'ENSEIGNANT QUI PEUT POURTANT PERMETTRE D'ENRÔLER LES ÉLÈVES DANS UNE TÂCHE}

Ainsi, si la posture épistémologique consistant à susciter un questionnement de la part des élèves et leur permettre de formuler des hypothèses quant à la réponse à certaines questions que se posent les économistes (même s'il ne s'agissait ici que de la définition d'un concept catégoriel) semble emporter l'adhésion des professeurs stagiaires de l'académie d'Aix-Marseille, sa mise en œuvre se révèle difficile. Ce type de démarche s'avère souvent « inconfortable » pour l'enseignant. En effet, par rapport à la situation dans laquelle on se contente de délivrer une définition que l'on demande ensuite aux élèves d'appliquer ou d'exemplifier, l'enseignant ne sait pas, dans une démarche de ce type, quels sont les arguments qui vont véritablement émerger dans la classe.

L'exercice est alors périlleux pour un professeur débutant dans le métier. Dans le cas qui nous occupe, la professeure stagiaire, sur la base d'une analyse $a$ priori, pensait que certains des élèves classeraient les chômeurs dans la population inactive, à partir d'une argumentation liée à l'activité au sens courant : le chômeur ne «fait rien ». A contrario, l'homme ou la femme au foyer « travaillent » (ils font « tourner la maison $»$ ).

En revanche, elle n'avait pas anticipé que le retraité puisse être classé comme actif, ou que le caractère « actif » du chômeur serait relié, par les élèves, à la « rémunération » touchée. Elle ne pensait ainsi pas voir surgir l'indemnisation du chômage.

Cela dit, on assiste à une prise de conscience de la part des élèves. Durant toute la séance commentée dans ce texte, les élèves se prennent véritablement au jeu de la compréhension des critères utilisés pour la définition de l'activité au sens économique du terme. Lorsque l'enseignante leur dit que l'économiste classe tel individu comme actif ou inactif alors qu'ils n'ont pas donné spontanément cette réponse, ils cherchent à trouver les critères qui sont à l'origine de ce classement.

À l'issue de la séance, tous (même Amélie !) ont compris certaines conventions à l'œuvre dans la définition de la population active. Ils savent que ce ne sont pas les allocations chômage qui permettent de classer un chômeur parmi les actifs, qu'un retraité est inactif malgré sa pension de retraite et qu'un homme ou une femme au foyer (malgré le flou évoqué concernant cet exemple), même s'ils travaillent dans un sens courant du terme, n'exercent pas une activité rémunérée (il aurait été préférable de préciser qu'ils n'occupent pas d'emploi et n'en cherchent pas) et sont donc classés parmi les inactifs.

Les difficultés rencontrées par Charlotte, travaillées en formation lors d'une séance d'allo-confrontation durant laquelle un groupe de pair réagit au montage de la séance filmée, sont analysées et discutées collectivement.

Cette séance de formation permet une prise de conscience : devenir observateur de son activité, prendre du recul par rapport à elle, conduit à se découvrir comme sujet agissant, qui fait des choix, prend des décisions et produit des objets qui auraient pu être différents. Aurait-il été possible de procéder autrement ? Le fait de laisser la parole aux élèves, initialement perçu comme une source d'inconfort professionnel, devient, grâce à l'exploration collective des gestes professionnels possibles, un moyen d'enrôlement de ces derniers dans la tâche.

L'étude, en formation, de cas similaires de difficultés rencontrées par des professeurs débutants, permet, progressivement, de construire une professionnalité et de rendre compatibles une conception épistémologique d'une discipline scolaire et une pratique d'enseignement. 


\section{NOTES}

1. Un autre article, publié en 2017 (Dollo, 2017), s'inscrit dans cette « recherche de faisabilité ». Il propose des analyses didactiques de séances mettant en œuvre un « temps de sensibilisation », étape de traitement d'une question des programmes de SES, introduite dans le préambule des programmes du cycle terminal au début des années 2010.

2. Selon une expression de Jeanine Bremond et Henri Lanta (1995).

3. Marcel Roncayolo est un géographe à qui le ministère de l'Éducation nationale confie (ainsi qu'à Guy Palmade, historien de l'économie) en 1965 la charge de définir ce que pourrait être une initiation aux faits économiques et sociaux destinée à des élèves de l'enseignement général.

4. En classe de Seconde, le chômage est présent dans le programme, à travers une question : « Le chômage : des coûts salariaux trop élevés ou une insuffisance de la demande ? » On retrouve ensuite la question du chômage en classe de Première, et en classe de Terminale, dans un thème intitulé "Travail, emploi, chômage ».

5. Il faut noter qu'il existe aujourd'hui des tentatives de prise en compte du travail non déclaré dans la mesure de la production.

\section{RÉFÉRENCES}

Amade-Escot, C. (2013). Potentialité de la Théorie de l'Action conjointe en didactique (TCAD) pour l'analyse des situations d'intervention en APS. Le Journal de la recherche sur l'intervention en éducation physique et sport (eJRIEPS), 30, 82-88.

Assude, T,. \& Mercier, A. (2007). Laction conjointe professeur-élèves dans un système didactique orienté vers les mathématiques. Dans G. Sensevy \& A. Mercier A. (dir.), Agir ensemble. L'action didactique conjointe du professeur et des élèves (p. 153-185). Rennes : PUR.

Astolfi, J.-P. (1993). Trois paradigmes pour les recherches en didactique. Revue française de pédagogie, 103, 5-18.

Bachelard, G. (1938/1983). La formation de l'esprit scientifique. Paris : Librairie Philosophique J. Vrin.

Bautier, E., \& Rochex, J.-Y. (1998). L'expérience scolaire des nouveaux lycéens, démocratisation ou massification? Paris : Armand Colin.

Bautier, E., \& Rochex, J.-Y. (2004). Difficultés d'apprentissage, processus de secondarisation et pratiques enseignantes : une hypothèse relationnelle. Revue Française de Pédagogie, 148, 89-100.

Beitone, A. (2005). Les chômeurs sont-ils des inactifs? Une énigme didactique. Skholê, Hors-Série $n^{0} 1$, 23-28.

Beitone, A., Cazorla, A., Hemdane, E. (2016). Dictionnaire de sciences économiques. Paris : Armand Colin.Beitone, A., Dollo, C., Hemdane, E., Lambert, J.-R. (2013). Les sciences économiques et sociales. Enseignement et apprentissages. Bruxelles : De Boeck. Bourdieu, P. (1992). Réponses. Paris : Seuil.

Bourdieu, P. (2001). Science de la science et réflexivité. Paris : Raisons d'agir.

Bourdieu, P., Chamboredon, J.-C., \& Passeron, J.-C. (1968). Le métier de sociologue. Préalables épistémologiques. Paris : Mouton-Bordas.

Brémond, J., \& Lanta, H. (1995). La pédagogie des sciences économiques et sociales : mythe fondateur ou réalité ? Dans P. Combemale (coord.), Les sciences économiques et sociales (p. 47-71). Paris : Hachette Éducation.

Brousseau, G. (1998). Théorie des situations didactiques. Grenoble : La pensée sauvage.

Bulletin officiel, $\mathrm{n}^{0} 21$ du 23 mai 2013.Chalmers, A. F. (1987). Qu'est-ce que la science ? Récents développements en philosophie des sciences : Popper, Kuhn, Lakatos, Feyerabend. Paris : La Découverte.

Dollo, C. (2001). Quels déterminants pour l'évolution des savoirs scolaires en Sciences Économiques et Sociales? (L'exemple du chômage) (thèse de doctorat en sciences de l'éducation, université de Provence, CIRADE). Aix en Provence. Accès en ligne : [https:// tel.archives-ouvertes.fr/tel-00006896/document].

Dollo, C., \& Johsua, S. (2002). Conceptions d'élèves et diversité des paradigmes en sciences économiques et sociales (l'exemple du chômage). L'Année de la Recherche en sciences de l'éducation, 2002, 181-208.

Dollo, C. (2003). Discussion et relativisme en Sciences économiques et sociales. Actes du colloque du CERFEE/LIRDEF, La discussion en éducation et en formation : socialisation, langage, réflexivité, identité, rapport au savoir et citoyenneté. Université Paul Valéry de Montpellier 3 et IUFM de Montpellier, 23-24 mai.

Dollo, C. (2005a). Les SES : épistémologie d'une discipline scolaire. Skholêe, Hors-série 1, 9-14.

Dollo C. (2005b). Généricité / spécificité d'un concept : La transposition didactique en sciences économiques et sociales. Revue Suisse des sciences de l'Éducation, 1, 85-102.

Dollo, C. (2009). Épistémologie et didactique en sciences économiques et sociales. De la recherche à la formation des enseignants. Recherche et formation, 60, 87-101. 
Dollo, C. (2017). Le « temps de sensibilisation » en sciences économiques et sociales : une vraie difficulté pour les enseignants entrant dans le métier. Quel dispositif de formation ? Éducation E Formation, e-307(02), 41-48.

Fichant, M. (1969). Sur l'histoire des sciences. Paris : Maspero.

Johsua, S., \& Dupin, J. J. (2003). Introduction à la Didactique des sciences et des mathématiques. Paris : PUF.

Marx, K. (1867-1883/1993). Le capital, livre 1. Paris : PUF.

Montoussé, M. (2004). La place des théories dans l'enseignement des sciences économiques et sociales. Idees, 138, 64-70.

Orange, C. (2003a). Débat scientifique dans la classe, problématisation et argumentation : le cas d'un débat sur la nutrition au cours moyen. ASTER, 37, 83-107.

Orange, C. (2003b). Apprentissages scientifiques, activités langagières et problématisation. Dans M. Jaubert, M. Rebière \& J. P. Bernié (dir.), Construction des connaissances et langage dans les disciplines d'enseignement. Actes du colloque pluridisciplinaire international (Bordeaux, du 3 au 5 avril 2003), IUFM d'Aquitaine, université V. Segalen Bordeaux 2.
Orange, C. (2008). Problématisation en sciences et dans les apprentissages scientifiques. Côté-Philo, 11, 39-47.

Schubauer-Leoni, M.-L. (1986). Maître-élève-savoir : analyse psychosociale du jeu et des enjeux de la relation didactique (Thèse de doctorat en sciences de l'éducation). Université de Genève.

Schubauer-Leoni, M.-L., Leutenegger, F., Ligozat, F., \& Fluckiger, A. (2007). Un modèle de l'action conjointe professeur-élèves : les phénomènes didactiques qu'il peut/doit traiter. Dans G. Sensevy $\&$ A. Mercier (dir.), Agir ensemble. L'action didactique conjointe du professeur et des élèves (p. 51-91). Rennes: PUR.

Sensevy, G. (2007). Des catégories pour décrire et comprendre l'action didactique. Dans G. Sensevy $\&$ A. Mercier (dir.), Agir ensemble: L'action didactique conjointe du professeur et des élèves (p. 13-34). Rennes: PUR.

Sensevy, G., \& Mercier, A. (dir.) (2007). Agir ensemble. L'action didactique conjointe du professeur et des élèves. Rennes: PUR.

Vatin, F. (2010). Marx et le travail : acte créateur et instrument d'aliénation. Revue du MAUSS permanente, 6 février 2010 [en ligne]. Repéré à : [http://www.journaldumauss.net/./?Marx-et-le-travail-acte-createur]. 